\title{
Addressing Privacy in a Federated Identity Management Network for E- Health
}

\author{
Liam Peyton, Jun Hu, Chintan Doshi, Pierre Seguin \\ School of Information Technology and Engineering, University of Ottawa \\ lpeyton@site.uottawa.ca,junhu@site.uottawa.ca,cdosh040@uottawa.ca \\ seguin_pierre@yahoo.ca
}

\begin{abstract}
E-health networks can provide integrated services to patients and health care workers that are more broadly accessible by leveraging Internet technology and electronic health records. However, issues of security and privacy must be addressed. In particular, compliance with relevant privacy legislation must be established. Federated identity management can enable users and service providers to securely and systematically manage identities and user profiles in a single sign on framework that controls access to personal information. In this paper, we use a simple ePrescription scenario to analyze the business and technical issues that need to be addressed in a Liberty Alliance federated identity management framework. We look at the potential impact of privacy compliance on three existing components of the framework (Discovery Service, Identity Mapping Service, Interaction Service) as well as a fourth component (Audit Service) that has been proposed to address potential privacy breeches in Liberty Alliance.
\end{abstract}

\section{Introduction}

E-health networks can provide more seamless and integrated services to patients and health care workers that are more broadly accessible by leveraging Internet technology and electronic health records. In order to do so, however, issues of security and privacy of personal health information must be addressed. In particular, it is important that compliance with the relevant privacy legislation is established.

Federated identity management can enable users and service providers to securely and systematically manage identities and user profiles in a single sign on framework that controls access to personal information. The Liberty Alliance project was established in 2001 as a consortium of technology vendors and consumer-facing enterprises to develop an open standard and set of specifications for federated identity management. A key concept in the Liberty Alliance project is a "Circle of Trust" (CoT), in which federated identity management is used to create a business to business (B2B) network of cooperating enterprises that provide integrated services to users. These cooperating enterprises have trust relationships and operational agreements established amongst them.

Health care networks by their very nature involve separate cooperating enterprises (general physician, hospital, pharmacy, lab, home care, etc.). Federated identity management is a mechanism that could be leveraged to secure personal health information across a heterogeneous network of services without requiring the coordination and assembly of a single central store of personal health information.

In this paper, we use a simple scenario based on an ePrescription service [4] to analyze the business and technical issues that need to be addressed in order to leverage the Liberty Alliance federated identity management framework. In particular, we look at the potential impact of privacy compliance on the ownership responsibilities and architecture associated with three existing components of the Liberty Alliance framework (Discovery Service, Identity Mapping Service, Interaction Service) as well as a fourth component (Audit Service) that has been proposed to address potential privacy breeches in Liberty Alliance [3].

\section{Background}

The European Union Directive on Privacy and Electronic Communication [8] is the leading example of comprehensive privacy legislation. In Canada, there is similar legislation known as the Personal Information Protection and Electronic Documents Act (PIPEDA) [17]. The United States is not as stringent, but does have similar legislation in the area of health 
care [10]. In Ontario, Canada, there is specific legislation for health information in the form of the Personal Health Information Privacy Act (PHIPA) [20] within the context of PIPEDA. PHIPA specifies the legal responsibilities of health information custodians in terms of how they are to handle personal health information. PHIPA aims to protect privacy and confidentiality of personal health information while establishing a set of rules for the collection, use and disclosure of that information.

A discussion of the issues and approaches to protecting information within federated identity management systems including the concept of a Circle of Trust (CoT) are detailed in [21]. A CoT is one in which an individual's identity and personal information is protected by a designated Identity Provider, while still allowing cooperating enterprises within the CoT to access and share the individuals personal information in a systematic manner that ensures the individual's permission is obtained and their identity protected. The importance of anonymity for end users to trust service providers was shown in [2] as an issue in early work on e-commerce adoption and privacy preferences, although this may be less true as Internet social interactions become more commonplace. A general discussion of identity management and privacy requirements is given in [15] including a discussion of anonymous identity versus pseudonymous identity. In the case of pseudonymous identity, one can link events across sessions to an identity, without actually knowing the identity or any identity data. Support of pseudonymous identity is central to how identity is protected in a Circle of Trust and is central to our analysis of a proposed Liberty Alliance audit trail service. In [9] the importance of audits for ensuring trust is established in relation to identity management and the concept of user "owned" data.

Early work on addressing the relationship between privacy enhancing technology and privacy legislation was done in [18], which proposed an Information Transfer Registry (ITR) to support the logging and auditing of information transfers between businesses in B2B networks. Key principles for compliance with privacy legislation were identified as:

1. Organizations must identify how they intend to use personal data and receive consent from the individual.

2. Organizations must establish internal procedures to document and safeguard their use of data.

3. Individuals must be given access to their data and have recourse to challenge its accuracy and use.
In analyzing potential privacy breeches in [3], it was proposed that an audit trail service within the Liberty Alliance framework would be a useful addition. It could be used to document that organizations are correctly implementing the first two principles so that an external entity could certify organizations within a CoT as compliant. At the same time, an audit trail service could be used to enable an individual to see how their data is being used and challenge its use in order to address the third principle.

Audit trails that record the details of user activity are relevant to privacy compliance. In [23], an extensible information security specification format acts as a compliance audit mechanism for enforcing business rules and information security policies based on audit trails. The design of a HIPAA compliant auditing system for automatically monitoring the data flow and the work flow of medical imaging system based on security requirements is outlined in [5]. Methods for logging events to an audit trail are well known with tools like AspectJ and Log4J. These tools make it possible to incorporate systematic logging with low overhead, even without modifying the existing code base of a system [6]. An approach to incorporating these into a managed service for monitoring performance and compliance is discussed in [19].

This paper focuses on the Liberty Alliance federated identity management framework, whose architecture is described in [22, 14], and whose approach to security and privacy is described in $[7,16]$. The ePrescription scenario that we use is adapted from the one presented in [4]. The main specifications that affect our analysis of the proposed audit trail service are the web services specification [14] and data services template [13]. The interaction service specification [1] is also relevant for securing the consent of the individual. The details of the discovery service [12] and identity mapping service [11] are also relevant to the manner in which the audit trail supports pseudonymous identity.

\section{Liberty Alliance e-Prescription Scenario}

Consider the following Liberty e-Prescription scenario which is illustrated in Figure 1. In a Liberty Alliance Circle of Trust, there is a prescription service, ePrescription that is used by doctors who write prescriptions, and patients who receive prescription drugs. In the CoT, prescriptions are sent to the patient's pharmacy, ePharmacy, for fulfillment and the pharmacy is able to bill the patient's insurance company, eInsurance. Throughout the scenario, the Identity Provider provides a single sign on (SSO) service for the CoT so that users need to authenticate 
or "log in" only once. After that, each service (ePrescription, ePharmacy, eInsurance) recognizes the patient by a different pseudonym (called "opaque identifier" in the Liberty Alliance literature) known only to them which is provided by the Identity Provider through an Identity Mapping service (IMS). When a service wishes to access data about a patient from another service, it first discovers the service which has the patient's data, using a Discovery Service (DS) within the Identity Provider to obtain an end point reference (EPR). The EPR contains security and identity tokens that allow the invoked service to extract their pseudonym or "opaque identifier" for the patient without revealing it to the calling service. The patient must have granted permission for the two services to share the data. If not, the Identity Provider can invoke an Interaction Service which can be used to contact the patient and obtain their permission.

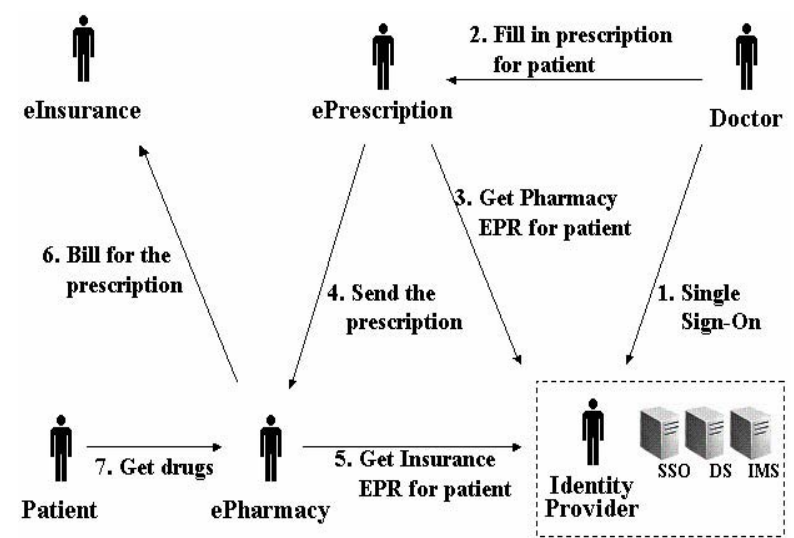

\section{Figure 1- Liberty Alliance e-prescription scenario}

Here is a detailed description of the steps involved in the scenario:

1. A Doctor is redirected to the Identity Provider to sign on to the CoT the first time they attempt to access any service in the CoT. This is a single sign on service ( $\mathrm{SSO}$ ) that authenticates the Doctor for access to any service in the CoT during the Doctor's online session.

2. The Doctor accesses the ePrescription service, selects one of his patients and enters a prescription for the Patient. The Doctor has permission to enter and see data about the Patient because the Patient has previously given permission for the Doctor to access their information. The Doctor is recognized by the ePrescription service based on the "opaque identifier" that is passed to it by the Identity Provider.
3. The ePrescrption Service communicates with a discovery service (DS) provided by the Identity Provider to obtain an end point reference (EPR) that will enable it to communicate with ePharmacy in order to fulfill the prescription on behalf of the Patient.

4. The ePrescription service sends the prescription information to the ePharmacy using the EPR. The EPR contains security and identity tokens from which ePharmacy can extract its pseudonym or "opaque identifier" for the Patient. Before ePharmacy accepts the prescription information, it must decide whether such a request is allowed based on Patient consent and its access control policies. If necessary, the patient's permission can be obtained through an interaction service (described in the next section).

5. The ePharmacy extracts its opaque identifier for the Patient from the EPR, and uses the discovery service (DS) of the Identity Provider in order to obtain another EPR that will enable it to invoke the Patient's eInsurance service.

6. The ePharmacy sends the billing information to eInsurance using the second EPR. The second EPR contains security and identity tokens from which eInsurance can extract its opaque identifier for the Patient. As in step 4, patient's permission must be obtained before sharing billing information.

7. The Patient identifies themselves to the ePharmacy (by authenticating with the Identity Provider) and receives their prescription drugs.

From this scenario, we can see that the Liberty Alliance Federated Identity Management Framework is able to protect identity through a federated system of pseudonyms supported by the Identity Management Service. It is also able to control the sharing of data and protect identity using end point references (EPR) provided by a discovery service, as well as obtain permission from the patient by the invocation of an Interaction Service. There is still a gap, though, as to whether the implementation of this control ensures privacy and complies with the relevant legislation.

\section{Privacy Compliance}

The ePrescription scenario described in Figure 1, can raise several privacy concerns about the way the service providers are using or sharing data. In identifying these concerns, we usually refer to a data service that allows personal data to be created, modified or viewed as an Attribute Provider, while the service accessing the Attribute Provider is a Web 
Service Client. For example, in Figure 1, eInsurance would be an Attribute Provider for prescription claims, while ePharmacy would be a Web Service client, registering its claims with eInsurance. Some possible privacy breaches in the scenario include. $[7,16]$ :

1. The Attribute Provider may fail to obtain the individual's consent before giving access to their data to a Web Service Client.

2. A malicious Attribute Provider may release an individual's attributes to an unauthorized Web Service Client.

3. The Attribute Provider may fail to properly enforce access-control and privacy policies.

4. A Web Service Client, on behalf of a user, obtains identity data, for unlawful purposes. For example, a doctor may be authorized to view a patient's medical data only under certain circumstances. If the doctor accesses patient's data for any other purpose (example: personal curiosity), the patient's privacy is breached.

Additional issues are identified in [3].

However, even in the absence of any privacy violations (malicious or otherwise), privacy legislation gives an individual the right to see and question how their data is being used. An audit trail can be used to provide this service as well as to provide verifiable evidence to a privacy office or regulatory body to certify compliance or investigate possible privacy breeches. For this reason, an audit trail service has been suggested for Liberty Alliance in [3]. Also [16] recognizes an audit mechanism as one of the privacyenabling functions for the ID-WSF framework. The audit service itself will not physically prevent privacy breaches from occurring but it can act as a deterrent and allow individuals, privacy officers, and regulatory bodies to monitor how data is being shared.

Figure 2, illustrates the situation and shows how the Attribute Provider could interact with the Discovery Service, Identity Mapping Service, and Interaction Service mentioned in the ePrescription scenario in section 3, as well as an Audit Trail Service in order to document what data sharing took place.

The steps are as follows:

1. A Web Service Client obtains an EPR from the Discovery Service, based on an encrypted opaque identifier maintained by the Identity Mapping Service, in order to interact with an Attribute Provider.

2. A request is made to the Attribute Provider. The Attribute Provider must decide whether or not the request is allowed according to its access control and privacy policies (referred to as a "policy decision point" and it must log the fact that the request took place in order to create an audit trail for verifying compliance.
3. The Attribute Provider interacts with the Discovery Service in the usual manner to obtain an EPR for the Interaction Service if needed, and an EPR for the Audit Service.

4. If permission from the Patient or the Doctor is required and has not yet been given, the Attribute Provider can make a request to the Interaction Service.

5. The Interaction Service can notify the Patient, interact with them and obtain their permission. Note that the Attribute Provider does not know the authenticated identity of the user or how to contact them. The Interaction Service is a special trusted service which has been given permission by the Patient to interact with them.

6. The Attribute Provider can then log the request with the Audit Trail Service.

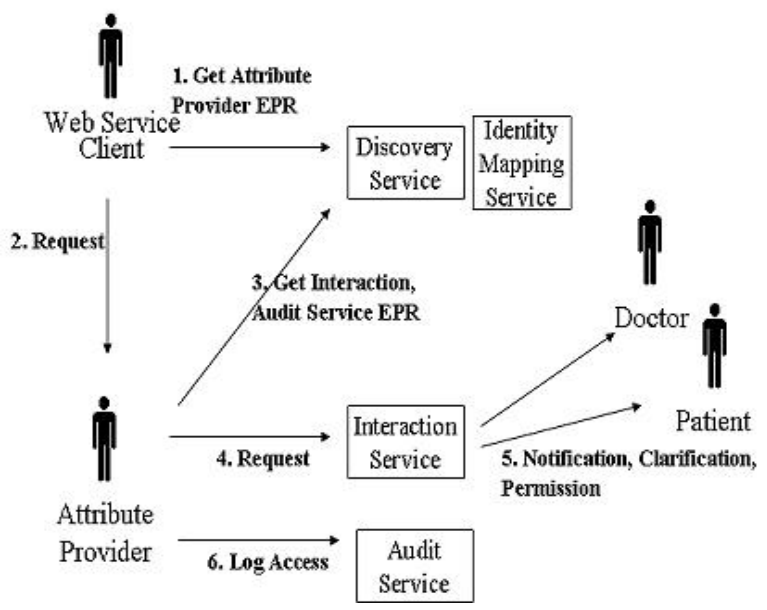

Figure 2 - Documenting data sharing for
privacy compliance

It is important to note some characteristics of this scenario that are relevant to the business control of the various services. It is reasonable to assume that the Discovery Service and Identity Mapping Service could be provided by a single trusted organization, the Identity Provider, since their operations are closely linked. In an e-health network, one could imagine a separate government agency or government regulated agency could be charged with safeguarding e-Identity.

In principle, the interaction service could be provided by the same organization. It is reasonable that the Identity Provider should have some means of contacting the individual whose identity they are managing. However, it is important for the Identity Provider to be arms length from the services being provided during the interaction. The Identity Provider is being trusted with safeguarding the identity of the individual and their identity information. Because of 
that sensitive role, the less personal health information it is aware of the better. It is when identity information is combined with personal health information that privacy can be compromised. As a result, one could imagine the individual might want a separate organization to provide their interaction service, or perhaps different organizations to provide different interaction services for different purposes.

It is essential that the Audit Service be provided by a different organization than the Identity Provider. The Audit Service can log requests from all Attribute Providers in the Circle of Trust. As well, the EPR used allows the Audit Service to recognize and correlate all data sharing requests related to a specific individual across all Attribute Providers without knowing the identity. This provides a comprehensive, consolidated audit trail of the individual's data and how it is being shared. This is important for privacy compliance. The single audit trail makes it straight forward for an individual to see who is using their data for what purpose as is required by privacy legislation. It also enables a privacy officer to document compliance or investigate alleged privacy breeches by validating the data sharing that occurs against the established policies and permissions of a given individual and the stated privacy practices of an Attribute Provider. However, if that information was ever combined with the identity information maintained by the Identity Provider it would be a complete breech of privacy. This is why the Audit Service must record the individual's audit trail in a privacy preserving manner, as explained in the next section

The Audit Service could be maintained by a privacy oversight organization, but it might also make sense for it to be maintained or at least accessible to an organization responsible for monitoring public health services and the quality of care provided. Privacy is just one aspect of quality of care.

\section{Audit Trail}

To see how an audit trail can provide complete documentation of data sharing for privacy compliance, we need to take a close look at the EPR used to communicate the request to the Attribute Provider that is logged. In Table 1, we define the fields of the event record that should be logged for a data sharing event in order to track privacy compliance and identify where the information for the field comes from.

The relevant security and identity tokens in an EPR for an Attribute Provider invocation that could be extracted and logged to the Audit Trail are the web service Client e.g. ePharmacy), InvocationID (e.g. the pharmacist fulfilling the prescription or possibly the doctor that wrote the prescription), TargetID (e.g. Patient the prescription is for) and the attribute Provider (e.g. eInsurance).

Table 1 - Audit trail event record

\begin{tabular}{|c|c|}
\hline Field Name & Description \\
\hline TargetID & $\begin{array}{l}\text { The Audit Service specific opaque } \\
\text { identifier extracted from the "target } \\
\text { identity" security token of the EPR. } \\
\text { E.g. the Patient. }\end{array}$ \\
\hline InvocationID & $\begin{array}{l}\text { The Audit Service specific opaque } \\
\text { identifier extracted from the } \\
\text { "invocation identity" token of the } \\
\text { EPR. E.g. the pharmacist or the } \\
\text { Doctor. }\end{array}$ \\
\hline Client & $\begin{array}{l}\text { Identifies the Web Service Client that } \\
\text { has queried the Attribute Provider. } \\
\text { The Attribute Provider (e.g. } \\
\text { eInsurance) would extract that from } \\
\text { the EPR passed to it by the Web } \\
\text { Service Client (e.g.ePharmacy), when } \\
\text { it accessed Patient's data and } \\
\text { eventually pass it to the Audit Trail. }\end{array}$ \\
\hline Provider & $\begin{array}{l}\text { Identifies the Attribute Provider that } \\
\text { is logging the message. This is a clear } \\
\text { text identifier ("recipient") located in } \\
\text { the EPR passed to the Audit Service. } \\
\text { (E.g. eInsurance). }\end{array}$ \\
\hline $\begin{array}{l}\text { Attribute } \\
\text { Name }\end{array}$ & $\begin{array}{l}\text { Attribute Providers share data based } \\
\text { on a data model [13] in the EPR in } \\
\text { which the names of attributes are } \\
\text { consistent across the CoT. The Web } \\
\text { Service Client (e.g. ePharmacy) } \\
\text { passes it to the Attribute Provider } \\
\text { (e.g. eInsurance) who passes it to the } \\
\text { Audit Service. }\end{array}$ \\
\hline Usage & $\begin{array}{l}\text { The Liberty ID-WSF architecture } \\
\text { supports a usage directive facility } \\
\text { [13]. This facility allows a Web } \\
\text { Service Client to state the purpose of } \\
\text { their request for data from an } \\
\text { Attribute Provider (and the Attribute } \\
\text { Provider can require it). The Attribute } \\
\text { Provider would pass it to the Audit } \\
\text { Service. }\end{array}$ \\
\hline Timestamp & Timestamp of the event. \\
\hline
\end{tabular}

Note that when communicating with the Audit Service, the Discovery Service is used to create a new EPR containing identity tokens for InvocationID and TargetID from which the Audit Service can extract the opaque identifiers which are unique to it. The Client 
and Provider are not "opaque" and can be passed directly.

Automated use of the "Usage" field requires more infrastructure than is covered in this paper. There must be agreement on specific values for "Usage", policy expressions that match values of this field, and association of "purpose" or "intended usage" information with requests for information.

\section{Analysis of Framework for E-Health}

The Liberty Alliance framework provides adequate infrastructure for protecting identity but an additional component, the Audit Service is needed to help support privacy compliance. The integration of the Audit Service into the Liberty Alliance Framework is reasonably straightforward. The Audit Service can be implemented as a standard Attribute Provider data service based on the relevant ID-WSF 2.0 specifications and templates.

The challenges are more organizational. The Circle of Trust must have a single well trusted organization that takes responsibility for the integrity of the Audit Trail Service, and that organization must be different from the organization responsible for the IDP. As well, each attribute provider must be convinced or required to $\log$ their events to the Audit Service. Methods for logging events to an audit trail are well known with tools like AspectJ and Log4J that make it possible to incorporate systematic logging with low overhead, even without modifying existing code [6]. Nonetheless mechanisms would have to be developed for each implementation technology used by different Attribute Providers although they would all be talking to the same web service interface.

In terms of ensuring privacy compliance, the Audit Service does not ensure privacy compliance but it is a useful tool to help track privacy compliance. It helps conform to privacy legislation by providing a historical record of data sharing events. This can be used to provide individuals with an account of how their data is being used and the ability to challenge it, as required by privacy legislation. It also provides the means for a Privacy Officer associated with the CoT or an outside regulatory agency to use the audit trail in conjunction with an inspection of the processes and policies in place at the CoT to validate compliance or investigate alleged breeches of privacy. More research is needed to investigate how this can be facilitated and supported more effectively.

With respect to privacy compliance for the Audit Trail Service itself, access control restrictions to the Audit Trail Service must be carefully considered and the individual's identity should not be easily deducible by a party having direct access to the audit trail records. It should be emphasized as well, that no attribute values are stored, just the names of the attributes.

For access control, Table 2 outlines the access control restrictions that should be placed on the audit trail records.

Table 2 - Access to the audit trail

\begin{tabular}{|l|l|l|l|l|}
\hline & Query & Add & Modify & Delete \\
\hline Individual & $\begin{array}{l}\text { X (own } \\
\text { data) }\end{array}$ & - & - & - \\
\hline $\begin{array}{l}\text { Attribute } \\
\text { Provider }\end{array}$ & - & X & - & - \\
\hline $\begin{array}{l}\text { Privacy } \\
\text { Officer }\end{array}$ & X & - & - & - \\
\hline
\end{tabular}

Attribute Providers should only be able to add records. Under no circumstances should it be possible to modify or delete records in the audit trail. The individual must have the right to query the audit trail records in which they are the PrincipalID. Privacy Officers or outside regulatory bodies will want to reserve the right to query any records. Since the Audit Service uses pseudonyms or opaque identifiers to persist its audit trail, the identity of individuals is protected. The opaque identifier used to identify the individual is meaningless to the Privacy Officer as long as they do not have access to the internal workings of the IDP. The individual, however, can have access to their records (and only their records), by going through the normal authentication process in which the IDP will pass the correct opaque identifier to the Audit Trail Service.

\section{References}

[1] Aarts R., Madsen P., eds., Liberty ID-WSF Interaction Service Specification, Ver. 2.0, Liberty Alliance Project, New Jersey, 2006. Accessed 2007/02. http://www.projectliberty.org/liberty/content/download/ 885/6231/file/liberty-idwsf-interaction-svc-v2.0.pdf

[2] Ackerman, M.S.; Cranor, L.F.; and Reagle, J. Privacy in e-commerce: Examining user scenarios and privacy preferences. In Proceedings of the ACM Conference on Electronic Commerce. New York: ACM Press, 1999, pp. $1-8$.

[3] M.Alsaleh, C. Adams, Enhancing Consumer Privacy in the Liberty Alliance Identity Federation and Web Services Frameworks. In Proceedings of the 6th Workshop on Privacy Enhancing Technologies (PET 2006), Cambridge, United Kingdom, June 2006

[4] T. Benthin, Liberty ePrescription Scenario ver. 1.1, Liberty Alliance Project, 2005.Accessed February 2007. http://www.projectliberty.org/liberty/adoption/healthcar e 
[5] Chen X.,Zhang J., Wu D., Han R., (2005) HIPPA's compliant Auditing System for Medical Imaging System, proceedings of the 2005 IEEE Engineering in Medicine and Biology 27th Annual Conference, Shanghai, China, Sept 1-4.

[6] J. Davies, N. Huismans, R. Slaney, S. Whiting, M. Webster and R. Berry, An Aspect Oriented Performance Analysis Environment, International Conference on Aspect Oriented Software Development, 2003. http://aosd.net/archive/2003/program/davies.pdf, Accessed 2007/02.

[7] Ellison, G., ed., Liberty ID-WSF Security Mechanisms; version 1.0-17, Liberty Alliance, Project, July 2003, http://www.projectliberty.org/resource_center/specificat ions/liberty_alliance_id_wsf_2_0_specifications, Accessed 2007/02.

[8] European Union Directive on Privacy and Electronic Communications. European Parliament, Brussels, Belgium, 2002. Accessed 2007/02. http://register.consilium.eu.int/pdf/en/02/st03/03636en2. pdf

[9] Gates, C., Slonim, J., Owner Controlled Information, New Security Paradigms Workshop 2003 Ascona Switzerland, ACM 2004.

[10] Health Insurance Portability and Accountability Act (HIPAA), United States Congress, United States, 1996. http://aspe.hhs.gov/admnsimp/pl104191.htm, Accessed February, 2007.

[11] Hodges, J., Aarts R., Madsen P , Cantor, S., Eds., Liberty ID-WSF Authentication, Single Sign-On, and Identity Mapping Services Specification Ver2.0, Liberty Alliance Project, New Jersey, 2006. http://www.projectliberty.org/liberty/content/download/ 871/6189/file/liberty-idwsf-authn-svc-v2.0.pdf, Accessed 2007/02.

[12] Hodges, J., Cahill, C., Eds., Liberty ID-WSF Discovery Service Specification. Ver2.0, Liberty Alliance Project, New Jersey, 2006. Accessed 2007/02. http://www.projectliberty.org/liberty/content/download/ 875/6201/file/liberty-idwsf-disco-svc-v2.0.pdf

[13] Kellomäki, S., Kainulainen, J., eds., Liberty ID-WSF Data Services Template ver.2.1, Liberty Alliance Project, New Jersey, 2006. Accessed 2007/02. http://www.projectliberty.org/liberty/content/download/ 879/6213/file/liberty-idwsf-dst-v2.1.pdf.

[14] Kemp, Y., eds., Liberty ID-WSF Web Services Framework Overview, Liberty Alliance Project, 2004, http://www.projectliberty.org/liberty/resource_center/pa pers, Accessed 2007/02

[15] Koch, M., and Möslein, K.M., Identity Management for Ecommerce and Collaborative Applications, International Journal of Electronic Commerce / Spring 2005, Vol. 9, No. 3, pp. 11-29.M.E. Sharpe Inc., 2005.

[16] Landau, S., eds., Liberty ID-WSF Security \& Privacy Overview; version 1.0, Liberty Alliance Project, 2003, http://www.projectliberty.org/resource_center/specificat ions/liberty_alliance_id_wsf_2_0_specifications. Accessed February 2007.

[17] The Personal Information Protection and Electronic Documents Act (PIPEDA), Department of Justice,
Canada, 2000. http://laws.justice.gc.ca/en/P8.6/text.html, Accessed 2007/02

[18] L.Peyton, M. Nozin, Tracking Privacy Compliance in B2B Networks, Sixth International Conference on Electronic Commerce, Delft, The Netherlands, October, 2004.

[19] L.Peyton, A. Rajwani, A Generative Framework for Managed Services, Third International Conference on Generative Programming and Component Engineering, Vancouver, October, 2004.

[20] PHIPA, Personal Health Information Protection Act, Government of Ontario, Canada, 2004. http://www.elaws.gov.on.ca/DBLaws/Statutes/English/04p03_e.htm, Accessed February 2007

[21] D. Shin, G-J Ahn, P Shenoy. Ensuring Information Assurance in Federated Identity Management, IEEE Intl. Conference on Performance, Computing, and Communications, 2004, p. 821-826

[22] Wason, T., eds., Liberty ID-FF Architecture Overview; version 1.2Liberty Alliance Project, March 2003. http://www.projectliberty.org/liberty/resource_center/pa pers, Accessed 2007/02.

[23] Yip, F. Ray, P. Paramesh, N. (2006) Enforcing Business Rules and Information Security Policies through Compliance Audits; XISSF - A Compliance Specification Mechanism, Business-Driven IT Management, BDIM '06,

[24] El Emam K, Jabbouri S, Sams S, Drouet Y, Power M, Evaluating Common De-Identification Heuristics for Personal Health Information, $\mathrm{J}$ Med Internet Res 2006;8(4):e28, http://www.jmir.org/2006/4/e28/ Accessed 2007/03. 